\title{
Evaluation of $\mathrm{CCK} 2$ receptor binding ligands: the inheritance of Thomas Behr
}

\author{
Otto C. Boerman • Luigi Aloj
}

Published online: 7 June 2011

(C) The Author(s) 2011. This article is published with open access at Springerlink.com

This issue of the European Journal of Nuclear Medicine and Molecular Imaging contains a series of studies comparing the in vitro and in vivo characteristics of a series of 12 cholecystokinin (CCK) receptor (CCKR) binding ligands [1-3]. Nine European research groups from Athens, Basel, Freiburg, Innsbruck, Ljubljana, London, Naples, Nijmegen and Rotterdam supported by a COST Action (BM0607) of the European Union have cooperated to carry out these studies. The project aimed to evaluate the in vitro and in vivo properties of 12 DOTAconjugated CCK2R ligands. The ligands were synthesized by the groups in Athens, Freiburg, Innsbruck, Nijmegen, London and Ljubljana and the following characteristics of the ligands were studied in standardized model systems:

- The affinity $\left(\mathrm{IC}_{50}\right)$ of the ligands for the CCK2R was determined in Berne

- The internalization kinetics of the ligands were determined in Naples and in Rotterdam

- The stability of the ligands was evaluated in Innsbruck

- The in vivo targeting properties of CCK2R-expressing tumours were evaluated in Nijmegen and in London

$\mathrm{CCK} /$ gastrin analogues were first proposed for radionuclide imaging and therapy of tumours approximately 15 years ago. The name of the late Thomas Behr is inextricably connected to the in vivo use of $\mathrm{CCK} /$ gastrin

O. C. Boerman $(\bowtie)$

Department of Nuclear Medicine, Radboud University Nijmegen

Medical Centre,

PO Box 9101, 6500 HB Nijmegen, The Netherlands

e-mail: O.Boerman@nucmed.umcn.nl

L. Aloj

Department of Nuclear Medicine, Istituto Nazionale Tumouri,

Fondazione "G.Pascale",

Naples, Italy analogues for in vivo targeting of CCK2R-expressing tumours (Fig. 1). Thomas Behr recognized the fact that excellent sensitivity of the pentagastrin test for detecting malignant $\mathrm{C}$ cells in patients with medullary thyroid carcinoma (MTC) suggested a high incidence of gastrin receptors in human MTC [4]. Indeed, at that time the autoradiography studies of Reubi et al. confirmed the expression of CCK/gastrin receptor in more than $90 \%$ of MTCs and in several other tumour types, such as small cell lung cancer, stromal ovarian cancers and astrocytomas [5].

At that time Thomas Behr worked at the Department of Nuclear Medicine at the Georg-August University in Göttingen headed by the late Prof. Wolfgang Becker. There he showed that a ${ }^{131} \mathrm{I}$-labelled truncated gastrin analogue accumulated specifically in CCK2R-expressing tumours in mice and men [4]. In a subsequent study Thomas Behr screened a series of 20(!) CCK/gastrin analogues [6]. All peptides were radioiodinated and the affinity for the CCK2R was determined and a few of these peptides were tested in nude mice with CCK2R-expressing xenografts. From these studies Thomas Behr selected minigastrin, a 12-amino acid peptide containing the pentaglutamic acid sequence as the optimal peptide for targeting CCK2R-expressing tumours.

Fig. 1 Thomas Behr: *21 May 1966- $\uparrow 20$ August 2010

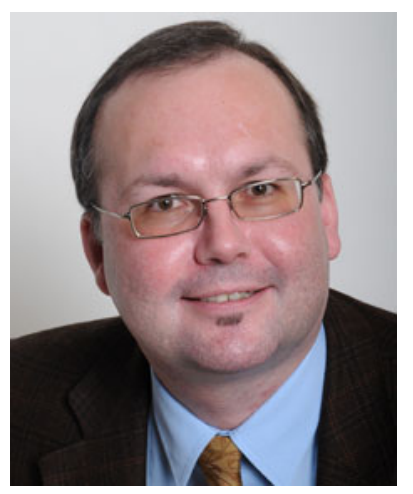


Table 1 Nomenclature, sequence and molecular weight of the 12 CCK2R binding ligands that were evaluated in the project

\begin{tabular}{|c|c|c|}
\hline Name & Sequence & MW (g/mol) \\
\hline G-CCK8 & DOTA-Gly-Asp-Tyr-Met-Gly-Trp-Met-Asp-Phe- $\mathrm{NH}_{2}$ & $1,507.0$ \\
\hline SA106 & DOTA-DAsp-Phe $\left(p-\mathrm{CH}_{2} \mathrm{SO}_{3} \mathrm{H}\right)$-HPG-Gly-Trp-HPG-Asp-Phe- $\mathrm{NH}_{2}$ & $1,482.6$ \\
\hline MG0 & DOTA-DGlu-Glu-Glu-Glu-Glu-Glu-Ala-Tyr-Gly-Trp-Met-Asp-Phe-NH ${ }_{2}$ & $2,037.0$ \\
\hline Sargastrin & DOTA-Gln-Gly-Pro-Trp-Leu-Glu-Glu-Glu-Glu-Glu-Ala-Tyr-Gly-Trp-Nle-Asp-Phe- $\mathrm{NH}_{2}$ & $2,483.6$ \\
\hline MG11 & DOTA-DGlu-Ala-Tyr-Gly-Trp-Met-Asp-Phe-NH ${ }_{2}$ & $1,531.0$ \\
\hline APH070 & DOTA-His-His-Glu-Ala-Tyr-Gly-Trp-Met-Asp-Phe-NH ${ }_{2}$ & $1,677.8$ \\
\hline PP-F10 & DOTA-DGln-DGln-DGln-DGln-DGln-DGln-Ala-Tyr-Gly-Trp-Met-Asp-Phe-NH ${ }_{2}$ & $2,042.9$ \\
\hline PP-F6 & DOTA-DGln-DGln-DGln-Ala-Tyr-Gly-Trp-Met-Asp-Phe--NH ${ }_{2}$ & $1,658.7$ \\
\hline PP-F16 & DOTA-DGln-DGlu-DGln-DGlu-DGln-DGlu-Ala-Tyr-Gly-Trp-Met-Asp-Phe-NH ${ }_{2}$ & $2,045.9$ \\
\hline PP-F11 & DOTA-DGlu-DGlu-DGlu-DGlu-DGlu-DGlu-Ala-Tyr-Gly-Trp-Met-Asp-Phe-NH ${ }_{2}$ & $2,048.8$ \\
\hline Cyclo-MG2 & DOTA-DGlu-(Ala-Tyr)-DLys-Trp-Nle-Asp-Phe- $\mathrm{NH}_{2}$ (cyclo DGlu-DLys) & $1,455.7$ \\
\hline MGD5 & DOTA-Gly-Ser-Cys-(Glu-Ala-Tyr-Gly-Trp-Nle-Asp-Phe- $\left.\mathrm{NH}_{2}\right)_{2}$ & $2,782.9$ \\
\hline
\end{tabular}

$H P G$ homopropargyl glycine

Thomas realized that for in vivo application these internalizing peptides should be labelled with a residualizing radiolabel and therefore, minigastrin was conjugated with DTPA and labelled with ${ }^{111}$ In and tested in xenografted nude mice and in a patient with MTC.

Ever since Thomas Behr carried out this pioneering work numerous research groups have synthesized new $\mathrm{CCK} /$ gastrin analogues in an attempt to further improve the tumour targeting properties. As part of the COST Action BM0607 on targeted radionuclide therapy we designed a set of in vitro and in vivo studies to select the peptides with optimal characteristics for in vivo targeting (Table 1). The group of Prof. Reubi in Berne, Switzerland determined the affinity of each of the 12 peptide analogues for the CCK2R using in vitro competitive autoradiography. This study showed that the $\mathrm{IC}_{50}$ of all ligands was in the nanomolar range $\left(0.2-3.4 \times 10^{-9} \mathrm{M}\right)$. The internalization kinetics of the 12 peptides as determined in Rotterdam and in Naples were also similar with most of the radiolabelled peptides internalized within $2 \mathrm{~h}$ [2].

A successful ligand for targeted radionuclide therapy should be stable in vivo at least during hours. Therefore the biological stability of the 12 analogues was studied in detail by the group in Innsbruck [3]. Variable stability in human serum was found for the different peptides with calculated half-lives between $4.5 \pm 0.1$ and $198 \pm 0.1 \mathrm{~h}$. In urine of normal mice only metabolized peptide fragments were detected even at short times after injection for all peptides. Matrix-assisted laser desorption/ionization time-of-flight mass spectrometry (MALDI-TOF MS) revealed a major cleavage site of all minigastrin derivatives between Asp and Phe- $\mathrm{NH}_{2}$ at the C-terminal end of the peptides.

The in vivo properties of the 12 radiolabelled peptides were studied in Nijmegen and in London. The Nijmegen group determined the biodistribution of the ${ }^{111}$ In-labelled peptides in mice with subcutaneous CCK2/gastrin receptorexpressing tumours in the flank and with receptor-negative tumours contralaterally [1]. The two CCK analogues both displayed relatively low tumour uptake as compared to the ten minigastrin analogues. Two linear minigastrin peptides displayed good accumulation in the CCK2 ${ }^{+}$tumour, but with very high kidney uptake. The linear analogue lacking the penta-Glu sequence (MG11) showed lower tumour uptake and also low kidney uptake. Varying the N-terminal Glu residues in the minigastrin analogues led to improved tumour targeting properties, with the analogue in which D-amino acids were used in the pentaglutamic acid sequence (PF11), displaying high tumour uptake in combination with low retention in the kidneys. Furthermore these studies showed that the cyclized peptide and the divalent peptide also showed high tumour to kidney ratios in this animal model. In addition, the group in London studied the in vivo tumour targeting properties of the 12 peptides by microSPECT/CT imaging. SCID mice with A431-CCK2R-positive and A431-CCK2R-negative tumours on the left and right shoulders, respectively, were injected with the ${ }^{111}$ In-labelled minigastrin/CCK peptide analogues radiolabelled with ${ }^{111}$ In to a specific activity of $30 \mathrm{MBq} / \mathrm{nmol}$. Whole-body SPECT/CT images after 1 and $4 \mathrm{~h}$ were obtained with a four-head microSPECT scanner equipped with 2-mm pinhole collimators. All images obtained showed preferential uptake in the CCK2Rexpressing tumours. Peptides were ranked based on the quantitation of tumour, kidney uptake and retention in the tumour during $4 \mathrm{~h}$. Four compounds appeared to have optimal properties for therapeutic application: the cyclic gastrin analogue, two minigastrin analogues (PP-F11 and PP-F16) and the dimeric compound MGD5.

In summary, in the studies described in this issue of the European Journal of Nuclear Medicine and Molecular 
Imaging the in vitro $[2,3]$ and in vivo [1] properties of a series of 12 CCK2R binding ligands were determined extensively. The studies indicated that three of these analogues have optimal characteristics for application in targeted radionuclide therapy of CCK2R-expressing cancers. In future studies the groups will further investigate the therapeutic potential of the selected compounds in mouse tumour models. Based on these studies a set of new ligands will be designed and synthesized. In addition, a working group aims to transfer one of these compounds for clinical studies in cancer patients. In conclusion, 15 years after the pioneering work of Thomas Behr, his gastrin analogues will be applied clinically for radionuclide imaging and possibly for radionuclide therapy.

Open Access This article is distributed under the terms of the Creative Commons Attribution Noncommercial License which permits any noncommercial use, distribution, and reproduction in any medium, provided the original author(s) and source are credited.

\section{References}

1. Laverman P, Joosten L, Eek A, Roosenburg S, Peitl PK, Maina T, et al. Comparative biodistribution of 12 (111)In-labelled gastrin/ CCK2 receptor-targeting peptides. Eur J Nucl Med Mol Imaging, doi:10.1007/s00259-011-1806-0.

2. Aloj L, Aurilio M, Rinaldi V, D'ambrosio L, Tesauro D, Peitl PK, et al. Comparison of the binding and internalization properties of 12 DOTAcoupled and (111)In-labelled CCK2/gastrin receptor binding peptides: a collaborative project under COST Action BM0607. Eur J Nucl Med Mol Imaging, doi:10.1007/s00259-011-1816-y.

3. Ocak M, Helbok A, Rangger C, Peitl PK, Nock BA, Morelli G, et al. Comparison of biological stability and metabolism of CCK2 receptor targeting peptides, a collaborative project under COST BM0607. Eur J Nucl Med Mol Imaging, doi:10.1007/s00259-011-1818-9.

4. Behr TM, Jenner N, Radetzky S, Béhe M, Gratz S, Yücekent S, et al. Targeting of cholecystokinin-B/gastrin receptors in vivo: preclinical and initial clinical evaluation of the diagnostic and therapeutic potential of radiolabelled gastrin. Eur J Nucl Med 1998;25(4):424-30.

5. Reubi JC, Schaer JC, Waser B. Cholecystokinin(CCK)-A and CCK-B/ gastrin receptors in human tumors. Cancer Res 1997;57(7):1377-86.

6. Behr TM, Jenner N, Béhé M, Angerstein C, Gratz S, Raue F, et al. Radiolabeled peptides for targeting cholecystokinin-B/gastrin receptor-expressing tumors. J Nucl Med 1999;40(6):1029-44. 